\title{
Bounded tracking controllers for the chaotic (forced) Duffing equation
}

\author{
A.A.J. Lefeber and H. Nijmeijer \\ Department of Applied Mathematics, \\ University of Twente \\ P.O.Box 217 \\ 7500 AE Enschede, The Netherlands \\ \{A.A.J.Lefeber,H.Nijmeijer\}@math.utwente.nl
}

\begin{abstract}
This paper deals with the design of feedback controllers for a chaotic dynamical system like the Duffing equation. Lyapunov theory is used to show that the proposed bounded controllers achieve global convergence for any desired trajectory. Some simulation examples illustrate the presented ideas.
\end{abstract}

\section{Introduction}

Starting with [7] controlling chaotic systems has recently become an extremely exciting and interesting research area, see the bibliography [1] and the paperscollection [8]. Basically, the method advocated by Ott, Grebogi and Yorke [7] consists of making small timedependent perturbations on available system parameters so that an initially chaotic system is steered towards any orbit contained in the chaotic attractor. The OGY-method, and more general, most literature on control of chaos, seems distinct from what is called control theory, although some clear connections exist.

The purpose of the present note is to work out, by means of a specific example, from a control theoretic point of view, a feedback control scheme that ensures the tracking of any desired trajectory under given input constraints. The example we consider is the controlled forced Duffing equation $[2,6]$ :

$$
\ddot{x}+p \dot{x}+p_{1} x+x^{3}=u+q \cos (\omega t)
$$

with $p \geq 0, p_{1}, q$ and $\omega$ constants and $u(\cdot)$ the (physical) control. For $u \equiv 0$, depending on the choice of the constants, it is known that solutions of (1) exhibit periodic, almost periodic, and chaotic behaviour; see e.g. [2]. Typically the control $u$ in (1) can be understood as a force (torque) applied to the uncontrolled forced Duffing equation. Clearly this also distinguishes the way controls appear from typical 'control of chaos' work like in the OGY-method, see [7], where control actions are generated via parameter perturbations. Notably, the differences with the OGY-method may be summarized as:

- The proposed state feedback controller achieves global tracking, i.e. no matter where the original system starts and no matter whether the uncontrolled system has a chaotic attractor or not.

- Tracking towards any desired trajectory is guaranteed; in particular the desired trajectory may not be an uncontrolled system trajectory, the prize being that a nonzero control action is needed to stay on the desired trajectory.

- The tracking is achieved by means of a state feedback controller, which automatically acts as an error correction mechanism.

The organization of this paper is as follows. In section 2 bounded feedback controllers, which are of composite form, are given. Simulations illustrating the control performance and tracking ability of the controllers are given in section 3 . Section 4 contains concluding remarks.

We conclude this introduction with some terminology and notation.

Let $\mathcal{F}$ denote the class of nondecreasing continuous differentiable functions $f: \mathbb{R} \rightarrow \mathbb{R}$ satisfying $f(0)=0$, $f^{\prime}(0)>0$ and $\sup _{x \in R}|f(x)| \leq 1$. This class is a subset of the class of saturating functions proposed in [3]. Examples of functions $f(x) \in \mathcal{F}$ are $f(x)=\tanh (x)$ and $f(x)=\frac{2}{\pi} \arctan (x)$.

Throughout we discuss the tracking problem under input constraints. Therefore we assume that the trajectory $x_{d}$ to track is bounded and has bounded first and second derivative, i.e. there exist positive constants $B_{0}$, $B_{1}$, and $B_{2}$ such that

$$
\left|x_{d}(t)\right| \leq B_{0},\left|\dot{x}_{d}(t)\right| \leq B_{1},\left|\ddot{x}_{d}(t)\right| \leq B_{2}, \quad t \geq 0
$$

A specific case which deserves separate attention, is formed by those desired trajectories that satisfy

$$
\left|\ddot{x}_{d}+p \dot{x}_{d}+p_{1} x_{d}+x_{d}^{3}-q \cos (\omega t)\right| \leq \mathcal{B}
$$

for some $B \geq 0$. Clearly, when $B=0, x_{d}$ is a trajectory of the uncontrolled system, and in this case our work is related to (but different from) various other control of chaos papers, see e.g. [8]. 


\section{State feedback control of the Duffing equation}

Assume we want the system (1) to follow any smooth desired trajectory $x_{d}(t)$, satisfying (2). For this purpose, we select the control via a state feedback law

$$
u=\ddot{x}_{d}+p \dot{x}_{d}+p_{1} x_{d}+x_{d}^{3}-q \cos (\omega t)-K_{p} e-K_{d} \dot{e}(4)
$$

where $e \equiv x-x_{d}, K_{d}$ and $K_{p}$ are positive constants, and $f_{1}, f_{2} \in \mathcal{F}$.

The controller (4) consists of two components, namely

1. the feedforward part: $\ddot{x}_{d}+p \dot{x}_{d}+p_{1} x_{d}+x_{d}^{3}-$ $q \cos (\omega t)$, which can be computed off-line,

2. the $\mathrm{P}$ (roportional) $\mathrm{D}$ (ifferential) feedback part: $K_{p} e+K_{d} \dot{e}$

The feedback terms in (4) are required to guarantee that the system converges towards $x_{d}(t)$. Once on this trajectory, the feedforward component keeps the system moving along it; note that this part equals zero if $x_{d}$ is a trajectory of the uncontrolled system.

The closed-loop system consisting of (1) and (4) is described by the time-varying second-order dynamics

$$
\ddot{e}+\left(p+K_{d}\right) \dot{e}+\left(p_{1}+K_{p}\right) e+e^{3}+3 e^{2} x_{d}+3 e x_{d}^{2}=0
$$

Proposition 1 Let $x_{d}(t)$ be any smooth bounded reference trajectory, satisfying (2). Then the state feedback controller (4) guarantees that (1) asymptotically converges towards $x_{d}(t)$, i.e.

$$
\lim _{t \rightarrow \infty}(e(t), \dot{e}(t))=(0,0)
$$

provided that $K_{p}>-p_{1}$ and $K_{d} \geq-p+2 B_{0} \sqrt{3}$.

Proof: We will use Lyapunov's direct method for obtaining our results, see for instance $[4,5,9]$.

Define $\lambda=\left(K_{d}+p\right) / 2$ and consider the candidate Lyapunov function

$$
V(e, \dot{e})=\frac{1}{2}(\dot{e}+\lambda e)^{2}+\frac{1}{2}\left(\lambda^{2}+p_{1}+K_{p}\right) e^{2}+\frac{1}{4} e^{4}
$$

which is positive definite. Along the closed-loop error dynamics (5), its time-derivative becomes:

$$
\begin{aligned}
\dot{V}(e, \dot{e})= & -\lambda \dot{e}^{2}-\left(3 x_{d} e+3 x_{d}^{2}\right) e \dot{e}- \\
& -\left(p_{1}+K_{p}+e^{2}+3 x_{d} e+3 x_{d}^{2}\right) \lambda e^{2}
\end{aligned}
$$

which is negative definite on $\Omega$ provided that

$$
\left(3 x_{d} e+3 x_{d}^{2}\right)^{2}-4 \lambda^{2}\left(p_{1}+K_{p}+e^{2}+3 x_{d} e+3 x_{d}^{2}\right)<0 .
$$

Since $\lambda \geq\left|x_{d}\right| \sqrt{3}$ and $\left(p_{1}+K_{p}+e^{2}+3 x_{d} e+3 x_{d}^{2}\right)=$ $p_{1}+K_{p}+\left(e+\frac{3}{2} x_{d}\right)^{2}+\frac{3}{4} x_{d}^{2}>0$ we obtain:

$$
\begin{aligned}
& \left(3 x_{d} e+3 x_{d}^{2}\right)^{2}-4 \lambda^{2}\left(p_{1}+K_{p}+e^{2}+3 x_{d} e+3 x_{d}^{2}\right) \\
& \leq\left(3 x_{d} e+3 x_{d}^{2}\right)^{2}-12 x_{d}^{2}\left(e^{2}+3 x_{d} e+3 x_{d}^{2}\right)- \\
& -4 \lambda^{2}\left(p_{1}+K_{p}\right)=-3 x_{d}^{2}\left(3 x_{d}+e\right)^{2}- \\
& -4 \lambda^{2}\left(p_{1}+K_{p}\right)<0
\end{aligned}
$$

Therefore, $\dot{V}(e, \dot{e})$ is negative definite which completes the proof.

When we know the initial error-state, we are able to determine an upperbound for the control law. Since (6) is a decreasing function along solutions of (5), it follows that for all $t \geq 0$ :

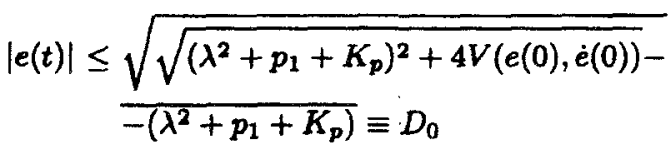

and

$$
|\dot{e}(t)| \leq \sqrt{4 V(e(0), \dot{e}(0))} \equiv D_{1}
$$

resulting in

$$
|u(t)| \leq \mathcal{B}+K_{p} D_{0}+K_{d} D_{1}
$$

This upperbound on the controller (4) depends on $V(e(0), \dot{e}(0))$, and the larger the initial errors are, the larger the upperbound will be.

To overcome this problem, we use a 'composite control law'. Apart from the tracking controller (4) we develop a bounded controller that globally controls the system into a specific area containing the origin. As soon as the system is in that area, we have bounds on both $e$ and $\dot{e}$, such that we can switch to the tracking controller (4). Since the first phase controller will be globally, i.e. it controls the system towards the specific area from any initial error state, the composite controller will be a bounded global asymptotically stable tracking controller.

Proposition 2 Consider the system (1) (with $p \geq 0$ ), together with the control law

$$
u=-q \cos (\omega t)-K_{p} f_{1}(x)-K_{d} f_{2}(\dot{x})
$$

with $K_{p} \geq 0, K_{d}>0$ and $f_{1}, f_{2} \in \mathcal{F}$. Then for all $C_{0}>0$ and $C_{1}>0$ there exists a $t_{s} \geq 0$ such that $|x(t)| \leq C_{0}$ and $|\dot{x}(t)| \leq C_{1}$ for all $t \geq t_{s}$, provided that $K_{p}$ is chosen large enough, i.e. $K_{p}>0$ for $p_{1}>0$ and

$$
K_{p}>\max _{x \in\left[-\sqrt{-p_{1}}, \sqrt{-p_{1}}\right]}-\frac{p_{1} x}{f_{1}(x)}
$$

otherwise.

Proof: The controller (10) results in the time-invariant closed-loop system

$$
\ddot{x}+p \dot{x}+p_{1} x+x^{3}+K_{p} f_{1}(x)+K_{d} f_{2}(\dot{x})=0 .
$$

Consider the radially unbounded candidate Lyapunov function

$$
V_{1}(x, \dot{x})=\frac{1}{2} \dot{x}^{2}+\frac{1}{2} p_{1} x^{2}+\frac{1}{4} x^{4}+K_{p} F_{1}(x)
$$

where $F_{1}(x)=\int_{0}^{x} f_{1}(\zeta) d \zeta$. This Lyapunov function is positive definite. Differentiating (12) along solutions of (11) yields

$$
\dot{V}_{1}(x, \dot{x})=-p \dot{x}^{2}-K_{d} \dot{x} f_{2}(\dot{x})
$$


which is negative semi-definite in the state $(x, \dot{x})$. Therefore, we have to determine the largest invariant set in $\left\{(x, \dot{x}) \in \mathbb{R}^{2} \mid \dot{V}_{1}(x, \dot{x})=0\right\}$, which is the origin. Application of LaSalle's theorem completes the proof.

Corollary 1 Consider the system (1). For all $C_{0}>\gamma$ and $C_{1}>0$, there exists a switching time $t_{s} \geq 0$ such that the composite control law

$$
u=\left\{\begin{array}{c}
-q \cos (\omega t)-K_{p, 1} f_{1}(x)-K_{d, 1} f_{2}(\dot{x}) \quad t<t_{s} \\
\ddot{x}_{d}+p \dot{x}_{d}+p_{1} x_{d}+x_{d}^{3}- \\
-q \cos (\omega t)-K_{p, 2} e-K_{d, 2} \dot{e} \quad t \geq t_{s}
\end{array}\right.
$$

results in a globally asymptotically stable closed-loop system, provided that $K_{d, 2}>-p+2 B_{0} \sqrt{3}$, $K_{p, 2}>-p_{1}, K_{p, 1}>\max _{x \in\left[-\sqrt{-p_{1}}, \sqrt{-p_{1}}\right]}-\frac{p_{1} x}{f_{1}(x)}$. Furthermore, there exists $a \beta>0$ such that the composite controller (13) satisfies $|u(t)| \leq \beta$ for all $t \geq 0$.

Proof: Let $t_{s}$ be a moment both $\left|x\left(t_{s}\right)\right| \leq C_{0}$ and $\left|\dot{x}\left(t_{s}\right)\right| \leq C_{1}$. From Proposition 2 the existence of $t_{s}$ follows and from Proposition 1 it follows that the second phase controller is asymptotically stable. Therefore the composite controller (13) is globally asymptotically stable.

Since $\left|x_{d}(t)\right| \leq B_{0}$ and $\left|\dot{x}_{d}(t)\right| \leq B_{1}$ for all $t \geq 0$, we have $\left|e\left(t_{s}\right)\right| \leq B_{0}+C_{0}$ and $\left|\dot{e}\left(t_{s}\right)\right| \leq B_{1}+C_{1}$, resulting in (9) for $t \geq t_{s}$. For the first phase we have $|u(t)| \leq$ $|q|+K_{p, 1}+K_{d, 1}$, therefore we have $|u(t)| \leq \beta$ for all $t \geq 0$ with

$$
\beta=\max \left\{|q|+K_{p, 1}+K_{d, 1}, \mathcal{B}+K_{p, 2} D_{0}+K_{d, 2} D_{1}\right\}
$$

It may be observed that the upperbound (14) is not accurate and thus possibly a better bound exists.

\section{Simulations}

To support our results, we simulated with MATLAB $^{T M}$ the system (1) with the controller (4). The Duffing parameters were selected as $p=0.4$, $p_{1}=-1.1, q=2.1$ and $\omega=1.8$, in which case the Duffing equation displays chaotic behaviour [2].

In our first simulation we initialize our system in $(x(0), \dot{x}(0))=(0,0)$ and the desired trajectory to track is an orbit of the uncontrolled system (i.e. $B=0$ ), initiated in $\left(x_{d}(0), \dot{x}_{d}(0)\right)=(0,1)$. To see clearly the difference between the chaotic uncontrolled motion and the regulated system, no control is applied during the first 30 seconds. At $t=30 \mathrm{~s}$ we initiate the composite controller (13) with $K_{p, 1}=5, K_{d, 1}=3, K_{p, 2}=5$ and $K_{d, 2}=7$. The switching moment $t_{a}$ is taken as the first moment $t$ at which both $|x(t)| \leq C_{0}=0.1$ and $|\dot{x}(t)| \leq C_{1}=0.1$. The resulting performance is depicted in Figure 1.
We can see in Figure 1a that within five seconds the system perfectly follows the desired trajectory. In Figure $1 b$ we can see the control-effort is initiated at $t=30$. At $t=32.6$ the state is small enough to switch to the second phase controller in order to establish tracking of the desired trajectory.

To show that we are able to track any desired trajectory, Figure 2 shows an anologous simulation, only our desired trajectory $x_{d}(t)$ has been taken as $x_{d}(t)=$ $\sin (t)$. We again see that within five seconds, our controller succeeds in tracking the desired trajectory, but, of course, a nonzero control-action in order to follow the desired trajectory is needed.

With the above parameter setting, we may compute, using Corollary 1 an upperbound for $u_{\max }$. For the first phase we have $|u(t)| \leq 5+3+2.1=10.1$. We switch to the second phase at a moment $t_{\text {a }}$ at which both $\left|x\left(t_{s}\right)\right| \leq 0.1$ and $\left|\dot{x}\left(t_{s}\right)\right| \leq 0.1$ such that in the first simulation we guarantee that $\left|e\left(t_{s}\right)\right| \leq 2.2$ and $\left|\dot{e}\left(t_{s}\right)\right| \leq 3.0$ (since $B_{0}=2.1$ and $B_{1}=2.9$ ). Therefore $V\left(t_{s}\right) \leq 110.5$ and so for all $t \geq t_{s}$ we have that $|e(t)| \leq$ 3.1 and $|\dot{e}| \leq 19.3$ implying that $|u(t)| \leq 151$; a very poor estimate, which in the simulations turns out to be about 15 times smaller.

The main regson for the poor estimate on the upperbound is caused by large $\left|e\left(t_{s}\right)\right|$. Therefore, a way to reduce the value of 151 is to switch at a moment that $\left|e\left(t_{s}\right)\right|$ is small, for instance as $\left|e\left(t_{s}\right)\right| \leq 0.1$. Notice we are only able to do this if the desired trajectory comes infinetely many times sufficiently close to the origin. The anology to the OGY-method seems obvious, although this propery not only holds for chaotic reference trajectories, but also for other signals. To illustrate this idea, Figure 3 shows the resulting performance when we redo our second simulation, tracking of $x_{d}(t)=\sin (t)$, but this time we switch as soon as both $|e(t)| \leq 0.1$ and $|\dot{e}(t)| \leq 3.0$. The resulting estimate for the upperbound on the control-effort can be calculated to satisfy $|u(t)| \leq 35.7$.

\section{Concluding remarks}

We have presented a bounded feedback controller for trajectory tracking in the forced Duffing equation. The methodology under which we have set up the controller (13) is subject to various modifications.

1. In the first stage of the composite controller we steer the system towards the origin; if the desired trajectory is far away from the origin it may become more profitable to steer the system with a slightly modified controller towards a point closer to the desired trajectory.

2. The switching time $t_{s}$ in the controller (13) is in some sense very important. Switching immedeately once the controlled system has reached the box where $\left|x\left(t_{s}\right)\right| \leq C_{0}$ and $\dot{x}\left(t_{s}\right) \mid \leq C_{1}$ may 
lead to relatively large control actions due to relatively large errors $\left(e\left(t_{0}\right), \dot{e}\left(t_{0}\right)\right)$. Thus it may be more appropriate to switch the controller at a later time for which $\left\|\left(e\left(t_{0}\right), \dot{e}\left(t_{0}\right)\right)\right\|$ is smaller.

3. The upperbound (14) is not tight and can certainly be improved. However in any case the main designer freedom in the composite controller (13) lies in the selection of the gains $K_{p, i}, K_{d, i}, i=1,2$ (and as mentioned the selection of $t_{s}$ ). The larger the gains, the faster the controller becomes.

4. Our controller is globally defined and the trajectory tracking was established for any initial state $(x(0), \dot{x}(0))$ and any desired trajectory. This was achieved via a careful Lyapunov analysis. Clearly, other Lyapunov functions may lead to other controllers and different convergence rates, but this is a usual problem of how to select a candidate Lyapunov function.

5. It is worthwhile to note that for the second part of the controller (13) the closedloop system satisfies $\dot{V} \leq-\alpha V$ for some $\alpha>0$ with $V$ given by (6). This implies that in this phase the controller guarantees exponential convergence towards the desired trajectory.

\section{References}

[1] G. Chen. Control and synchronization of chaotic systems (a bibliography), 1997. available from $\mathrm{ftp}: / / \mathrm{ftp}$.egr.uh.edu/pub/TeX/chaos.tex (login name "anonymous", password: your email address).

[2] G. Chen and X. Dong. On feedback control of chaotic continuous-time systems. IEEE Transactions on Circuits and Systems I: Fundamental Theory and Applications, 40(9):591-601, 1993.

[3] Y. Chitour, W. Liu, and E. Sontag. On the continuity and incremental-gain properties of certain saturated linear feedback loops. International Journal of Robust and Nonlinear Control, 5:413-440, 1995.

[4] W. Hahn. Stability of Motion. Springer-Verlag, Berlin, 1967.

[5] H.K. Khalil. Nonlinear Systems. Macmillan, New York, 1992.

[6] H. Nijmeijer and H. Berghuis. On Lyapunov control of the Duffing equation. IEEE Transactions on Circuits and Systems I: Fundamental Theory and Applications, 42(8):473-477, 1995.

[7] E. Ott, C. Grebogi, and J. Yorke. Controlling chaos. Physical Review Letters, 64(11):1196-1199, 1990.

[8] E. Ott, T. Sauer, and J. Yorke, editors. Coping with chaos. Wiley, 1994.

[9] M. Vidyasagar. Nonlinear Systems Analysis. Prentice Hall, Englewood Cliffs, second edition, 1993.
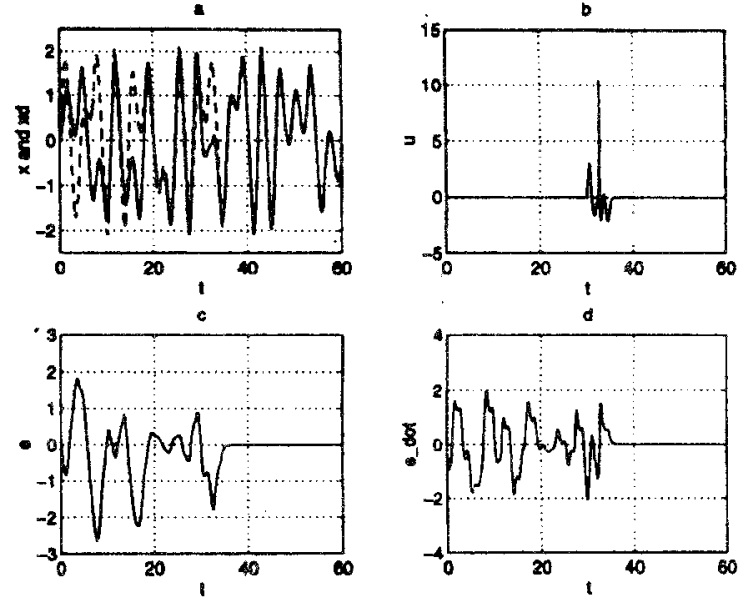

Figure 1: $x_{d}$ solution of Duffing, $K_{p, 1}=K_{p, 2}=5, K_{d, 1}=$ $3, K_{d, 2}=7$.
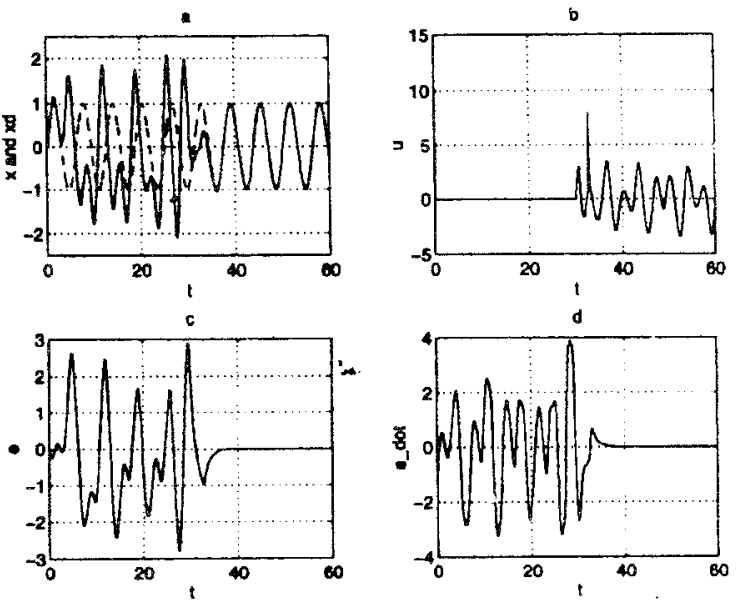

Figure 2: $x_{d}(t)=\sin (t), K_{p, 1}=K_{p, 2}=5, K_{d, 1}=3$, $K_{d, 2}=7$.
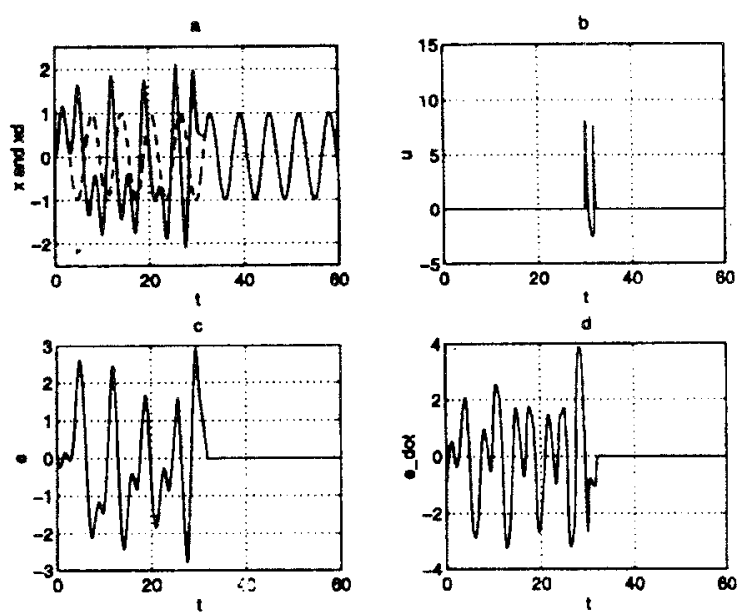

Figure 3: $x_{d}(t)=\sin (t), K_{p, 1}=K_{p, 2}=5, K_{d, 1}=3$, $K_{d, 2}=7$, OGY-like switching strategy. 